\title{
DETERMINAÇÃO DAS PROPRIEDADES FÍSICO-QUÍMICAS DE FLUIDOS DE PERFURAÇÃO EM TEMPO REAL EM UMA UNIDADE DE ESCOAMENTO DE FLUIDOS
}

\author{
S. C. MAGALHÃES FILHO ${ }^{1}$, E. V. N de NORONHA ${ }^{1}$, L. A. CALÇADA ${ }^{1}$, C. M. SCHEID ${ }^{1}$, H. \\ L. S. DE ALMEIDA ${ }^{2}$, C. H. DE SÁ ${ }^{3}$, M. FOLSTA ${ }^{3}$ \\ ${ }^{1}$ Universidade Federal Rural do Rio de Janeiro, Instituto de Tecnologia, Departamento de \\ Engenharia Química, Laboratório de Escoamento de Fluidos Giulio Massarani \\ ${ }^{2}$ Universidade Federal do Rio de Janeiro, Centro de Tecnologia da UFRJ. \\ ${ }^{3}$ Centro de Pesquisa Leopoldo Américo Miguez de Mello (CENPES) \\ E-mail para contato: sergiomagalhaes@sergiomagalhaes.eng.br
}

\begin{abstract}
RESUMO - Frente aos atuais cenários de perfuração de poços de petróleo, novas tecnologias vêm sendo desenvolvidas para comporem um sistema de soluções necessário para vencer os desafios técnicos. Janelas operacionais estreitas (faixa de pressão na qual o sistema de bombeio deve ser mantido) são um dos principais desafios à automação do sistema de perfuração, neste sentido, antever possíveis problemas operacionais é fundamental. Este trabalho tem o objetivo de avaliar sensores que aferem em tempo real as propriedades físico químicas dos fluidos de perfuração. Sendo estas aferições realizadas diretamente na linha de processo fica possibilitado um diagnóstico mais rápido do estado do fluido, assim como se torna possível uma construção de malha de controle para a correção de seu estado, se necessário. Por meio da comparação direta entre medidas de processo e laboratoriais de referência, ficou demonstrado que é possível se automatizar as medidas físico químicas dos fluidos de perfuração. Alguns sensores comerciais estão prontos para uso, outros necessitaram ser modificados e dois foram totalmente desenvolvidos.
\end{abstract}

\section{INTRODUÇÃO}

Este trabalho objetivou desenvolver uma malha sensorial capaz de aferir, em tempo real, as medidas físico químicas de fluidos de perfuração. Tais medidas atualmente na indústria são feitas em instrumentos de bancada, de forma manual. Para tanto, uma unidade experimental de escoamento de fluidos com alto nível de automação foi desenvolvida, onde os sensores foram instalados. Esta unidade é capaz de produzir fluidos de perfuração e bombeá-los por linhas de escoamento, sendo possível o controle remoto da pressão, temperatura e vazão. A malha sensorial atualmente é capaz de medir reologia, densidade, condutividade e estabilidade elétrica em tempo real. 


\title{
2. MATERIAL E MÉTODOS
}

\subsection{Unidade automatizada de escoamento de fluidos}

2.1.1. Estrutura Física: Para a avaliação da malha sensorial, uma unidade de escoamento de fluidos foi construída, cujas principais funções foram oferecer uma infraestrutura para fabricar fluidos de perfuração com rigor técnico e prover meios de simular perturbações semelhantes àquelas encontradas nos campos de perfuração. Na Figura 1 é demonstrado o esquema mais recente da unidade de escoamento de fluidos.

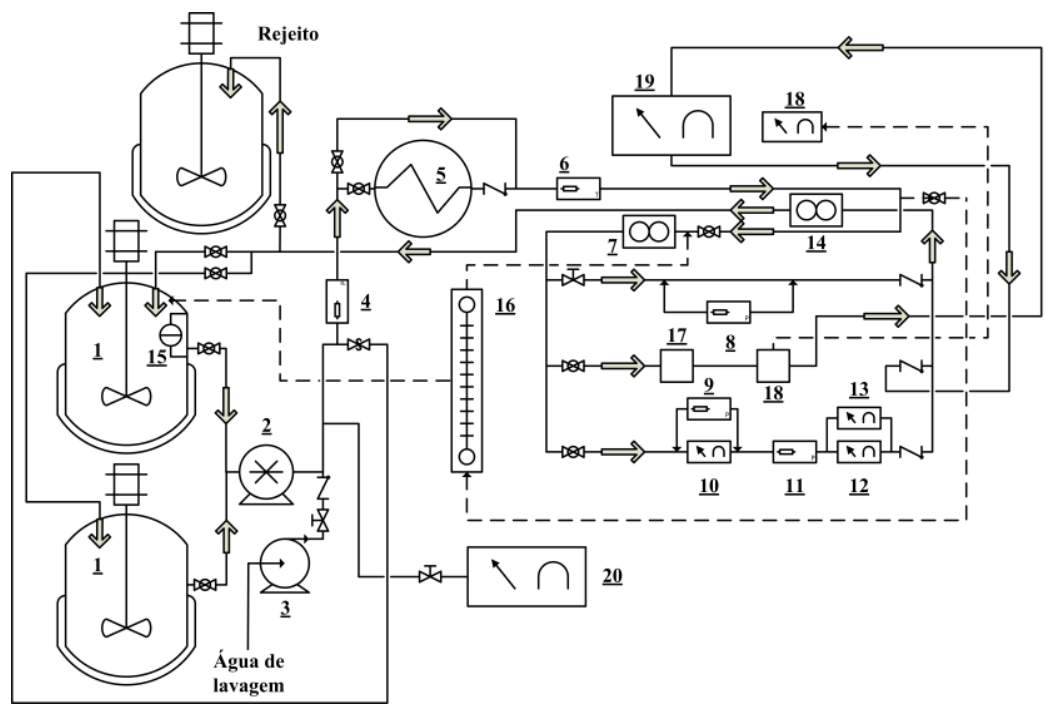

\author{
$1=$ Tanques de mistura com controle PID \\ $2=$ Bomba helicoidal com controle PID \\ $3=$ Bomba centrífuga com controle $\mathrm{ON} / \mathrm{OFF}$ \\ 4, $11=$ Medidores de pressãor \\ $5=$ Trocador de calor \\ $6=$ Medidor de temperatura \\ $7=$ Medidor de vazão volumétrica \\ 8, 9=Medidores de pressão diferencialr \\ $10=$ Reômetro de processo \\ 12=Condutivímetro de processo \\ 13=Medidor de estabilidade elétrica de processo \\ 14=Densímetro de processo \\ 15=Medidor de nível \\ $16=$ Simulador de fraturas \\ 17=Medidor de concentração de sólidos \\ 18=Analisador de tamanhos e contador de \\ partículas de processo \\ 19=Medidor de teor de água em óleo de processo \\ 20=Célula de filtração de processo
}

Figura 1 - Desenho esquemático da unidade de escoamento de fluidos automatizada

A unidade é capaz de produzir até 1000 litros de fluido de perfuração por batelada, podendo ser tanto fluidos a base de água quanto fluidos a base de óleo. Possui sistema de bombeio tanto para o fluido de perfuração quanto para água de limpeza; sensores de pressão, de temperatura, de vazão e sensores de nível nos tanques; possui um trocador de calor para aquecimento e resfriamento; válvulas automáticas para direcionamento de fluxo dentre outros sensores. Além destes, abriga os sensores específicos para leitura das propriedades de interesse dos fluidos de perfuração, que são: sensor para reologia, para densidade, para condutividade elétrica e para estabilidade elétrica.

2.1.2. Estrutura Virtual: Como centro de comando e processamento de dados foi construído um software com as funções de ser o supervisório, o controlador e o armazenador de todas as informações que trafegam na rede analógica montada para gerenciar os equipamentos. $\mathrm{O}$ software foi construído em linguagem Labview. O mesmo é capaz de receber todos os dados gerados pelos instrumentos, efetuar os cálculos convertendo os sinais analógicos nas variáveis de interesse e armazenar as informações em um banco de dados e reenviar os sinais de controle para bomba, agitador, etc. 


\subsection{Equipamentos laboratoriais, de processos, e suas metodologias}

2.2.1. Reologia: $\mathrm{O}$ equipamento laboratorial atualmente usado pelas indústrias é o viscosímetro de bancada da FANN Instruments, modelo 35A. Este viscosímetro utiliza o princípio dos cones concêntricos, conhecido como Couette, sendo capaz de fornecer não somente viscosidade, como também tensão de cisalhamento e taxa de deformação. Devido a sua possibilidade de controle de rotação, determinando a tensão em até seis pontos diferentes de taxa, o FANN 35A pode ser usado para caracterização reológica de fluidos de perfuração, os quais apresentam comportamento pseudo plástico. A resposta típica desse instrumento é a medida de tensão cisalhante em seis diferentes velocidades de medição, respectivamente a 3, 6, 100, 200, 300 e 600 RPM, que correspondem a 5,1, 10,2, 170, 340, 511 e 1021 1/s, respectivamente (Fann Instruction Manual model 35A, 2009).

O instrumento de processo utilizado para aferição da reologia em tempo real foi o viscosímetro da BROOKFIELD, modelo TT-100. Este viscosímetro utiliza o mesmo princípio do viscosímetro de bancada, com mudança manual das velocidades. Para reverter esta característica manual, foi proposta uma modificação substituindo o sistema original do motor do fabricante por um sistema computador/servoconversor/servomotor. Desta forma, o sistema proposto é totalmente automatizado e as informações de taxa de deformação agora são geradas diretamente no software criado. Na Figura 2 podem ser observados os equipamentos descritos acima.

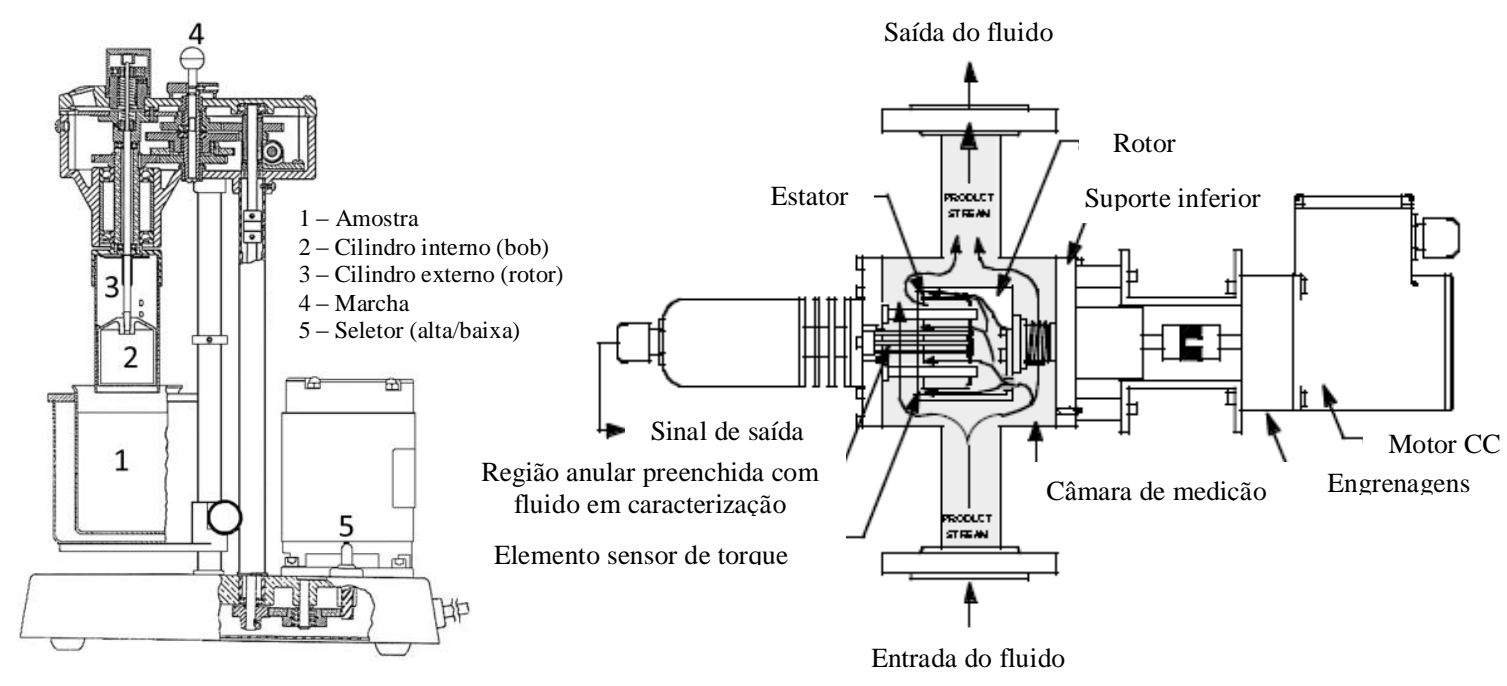

Figura 2 - À esquerda o viscosímetro FANN 35A (adptado de Fann Instruction Manual Model 35A, 2009), à direita o viscosímetro da BROOKFIELD, modelo TT-100 (adptado de Brookfield Instruction Manual and Guides Model TT-100, 1993).

2.2.2. Densidade: $\mathrm{O}$ equipamento de bancada possui uma escala graduada, onde é feita a leitura de densidade depois que o equilíbrio entre o peso da amostra e o peso deslizante do equipamento é atingido (Fann Instruction Manual Model 141 Mud Balance, 2013). Para medidas em tempo real foi utilizado um medidor de vazão mássica, do tipo Coriolis, especialmente 
aperfeiçoado pelo fabricante para aferições de densidade.

2.2.3. Condutividade e estabilidade elétrica: Este par de medidas é usado para verificar o estado dielétrico de fluidos de perfuração a base de óleo. Os fluidos a base de óleo são emulsões entre fases oleosas e aquosas. O monitoramento do estado dielétrico norteia a qualidade da emulsão, baixos valores significam que existe água 'fracamente' blindada, ou seja, a adsorção das gotículas de água não está satisfatória. Esse preceito é válido porque a fase dispersa é água (polar), e a contínua é o óleo (apolar) (Schramm, 2000). O teste consiste em aplicar uma tensão alternada a $340 \mathrm{~Hz}$ entre dois eletrodos submersos no fluido. À medida que a tensão aumenta, a intensidade da corrente que trafega também o faz, até que esta última atinja 61 micro amperes. Neste ponto é feita a aferição da voltagem necessária para conduzir esta corrente (Fann Instruction Manual, Model 23D, 2013). Caso o fluido se torne instável e a emulsão se quebre, a reologia mudará drasticamente, perdendo-se o controle das pressões do poço. Para as medidas de estabilidade elétrica em tempo real foi desenvolvido um sistema de medição. O sistema é capaz de reproduzir tecnicamente os efeitos causados pelo FANN 23D, além de possuir outras versatilidades como monitoramento da voltagem e corrente em tempo real, manipulação de parâmetros elétricos como tipo de onda elétrica, frequência, amplitude da rampa de ascensão da voltagem, dentre outras.

\section{RESULTADOS E DISCUSSÕES}

\subsection{Obtenção da reologia}

Na Figura 3, são apresentados os resultados típicos para a reologia aferida no TT-100 e FANN 35A. O gráfico da esquerda demonstra os resultados para uma amostra de glicerina, e à direita para um fluido de perfuração a base de água, sem sólidos em suspensão.
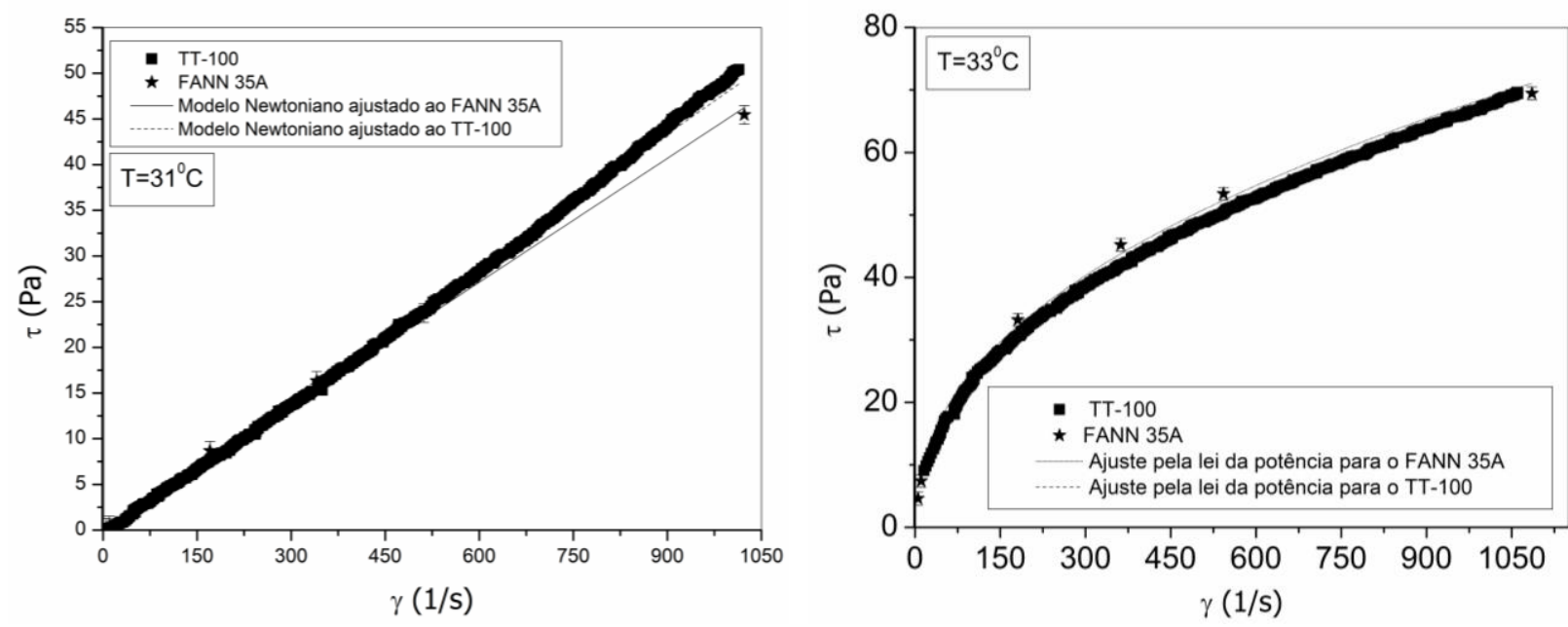

Figura 3 - Dados de tensão cisalhante em função da taxa de deformação. À esquerda, uma solução de glicerina, à direita, uma solução polimérica. 
Para ambos os casos, foram encontrados convergências nas medidas de processo e de bancada. Na Figura 4 são demonstrados resultados típicos para fluidos de perfuração com sólidos em suspensão, tanto a base de água, à esquerda, quanto à base de óleo, à direita.
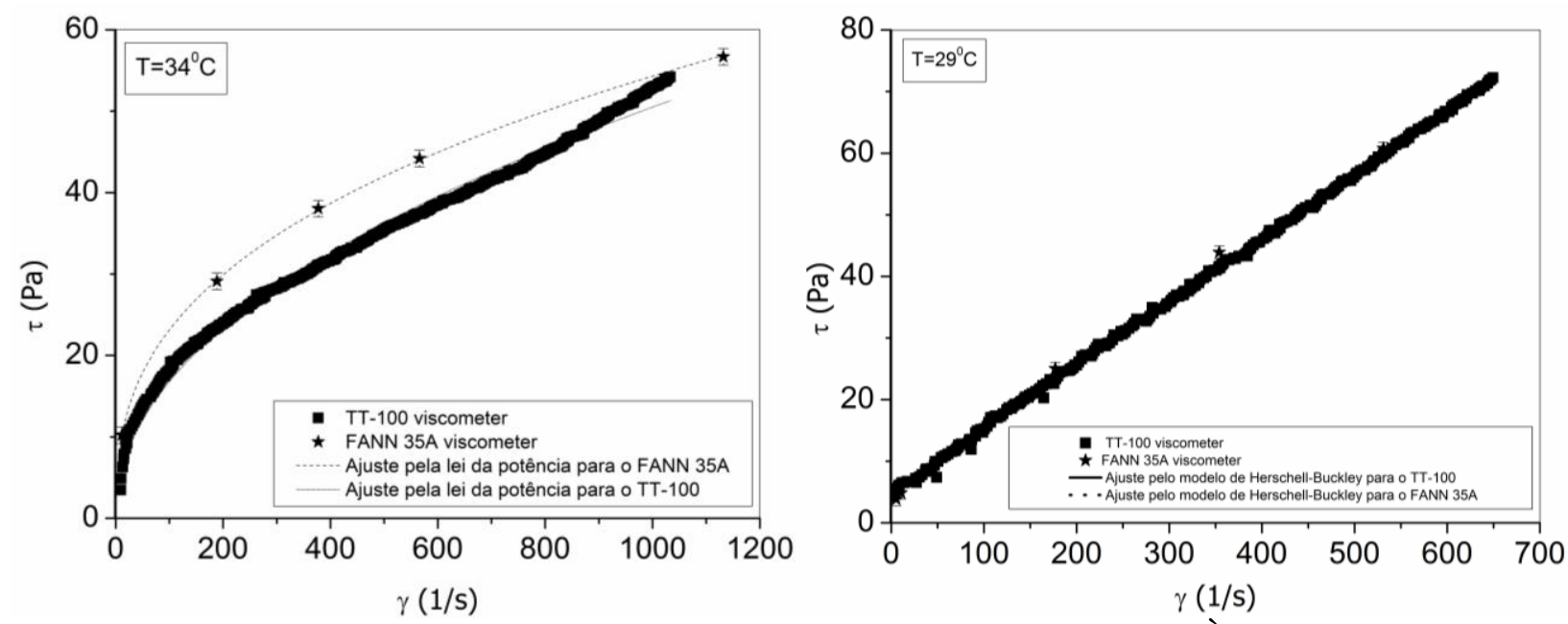

Figura 4 - Dados de tensão cisalhante em função da taxa de deformação. À esquerda, um fluido de perfuração a base de água, à direita, um fluido de perfuração a base de óleo.

Para o fluido a base de óleo, na faixa estudada, houve convergência nas medições de bancada e processo, já para os fluidos aquosos, divergências são encontradas entre as medidas. A literatura afirma que fluidos com alta concentração de sólidos em suspensão tendem a ser mais difíceis de caracterizar, pois os sólidos causam um escorregamento na parede do cilindro interno, aumentando as imprecisões nas medidas. Como o espaço anular (razão entre raios) do TT-100 é menor do que do FANN 35A (FANN=1,0678, TT-100=1,0481), e quanto menor este espaço mais pronunciado é o efeito dos sólidos sobre a medição. Acredita-se que esta é a razão destas divergências (Barnes, 2000).

\subsection{Obtenção da densidade}

Os testes de densidade consistiram em preparar um fluido de perfuração base nos tanques, sem sólidos em suspensão, e adensá-los controladamente com barita ou calcário. A cada alteração no valor da densidade, uma amostra era retirada do processo e aferida no instrumento de bancada de referência. Um resultado típico de adensamento pode ser observado na Figura 5. À esquerda está o resultado para o adensamento de um fluido a base de água, e à direita para um fluido à base de óleo. 

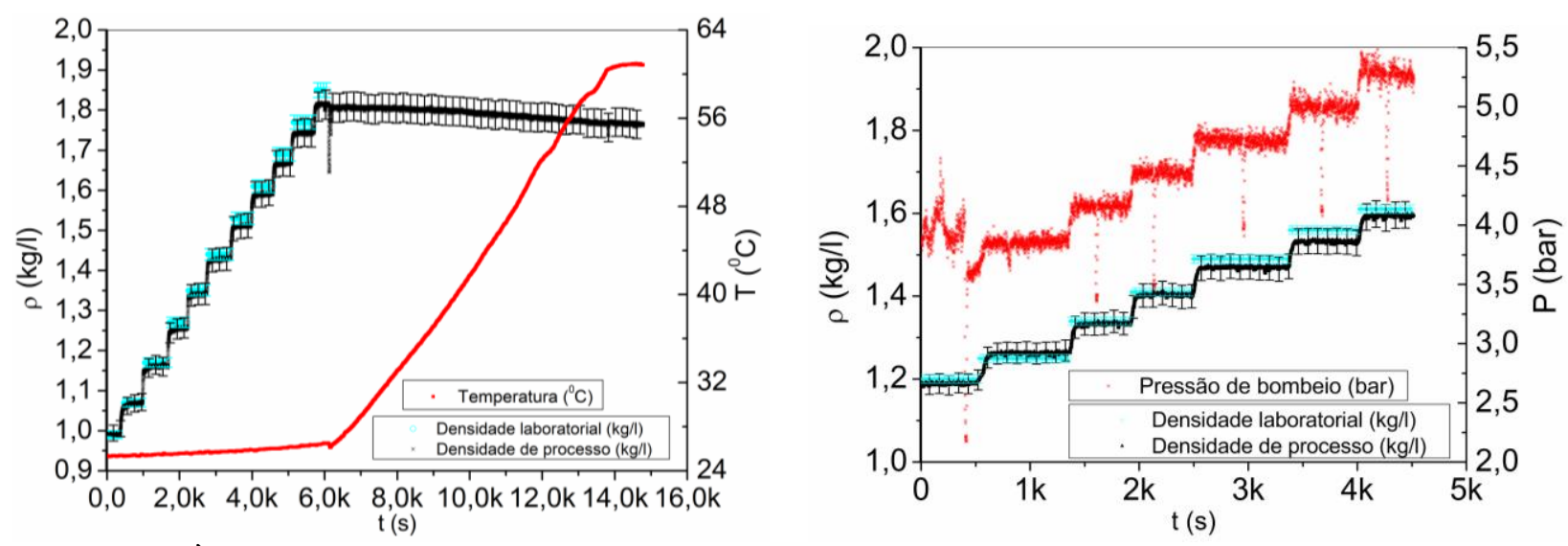

Figura 5 - À esquerda dados de densidade e temperatura por tempo de teste, à direita dados de densidade e pressão do sistema por tempo de teste, ambos durante adensamento.

Observando a Figura 5, em ambos os casos, verifica-se que houve similaridade nas aferições feitas na balança de lama (dados em azul) e as medidas do densímetro de processo (preto). As barras verticais representam os erros experimentais associados à imprecisão sensorial. Também durante o adensamento com o fluido a base de água foi feito um aquecimento (dados em vermelho) para verificar a autocorreção do densímetro com a temperatura. Como a balança de lama não possui controle térmico, somente as medidas de processo foram adquiridas.

\subsection{Obtenção da condutividade e estabilidade elétrica}

O protótipo e o FANN 23D foram colocados lado a lado na bancada para aferições em amostras padrão. Uma amostra apresentou estabilidade elétrica típica (acima de 300V), e outra, com alta concentração de água, valores abaixo de 200V. Na Figura 6 podem ser observados os resultados preliminares.
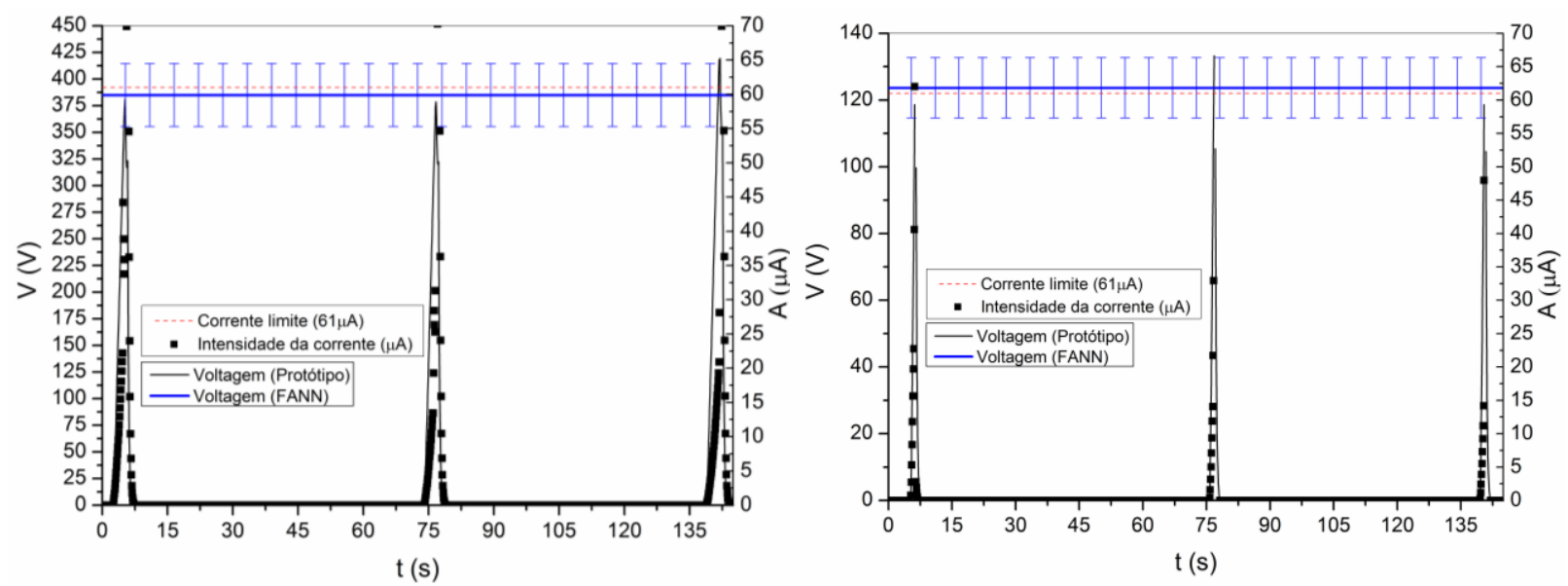

Figura 6 - Dados de voltagem e intensidade de corrente em função do tempo de teste. 




19 a 22 de outubro de 2014

Florianópolis/SC

Na Figura 6 as linhas contínuas são os valores de voltagem aferidos no protótipo, os pontos são os valores de intensidade de corrente, também aferidos pelo protótipo. A linha contínua em azul, horizontal, é a medida de voltagem aferida no FANN 23D. A linha tracejada vermelha é a corrente máxima que o sistema deve atingir antes de seu desligamento. Os três picos representam o teste em triplicata. É possível verificar que as medidas de voltagem do protótipo (picos das linhas pretas) estão dentro do erro experimental das medidas do equipamento de referência, em ambas as amostras. O próximo passo foi testar o sensor inserindo-o no processo. Na Figura 7 são demonstrados os resultados.
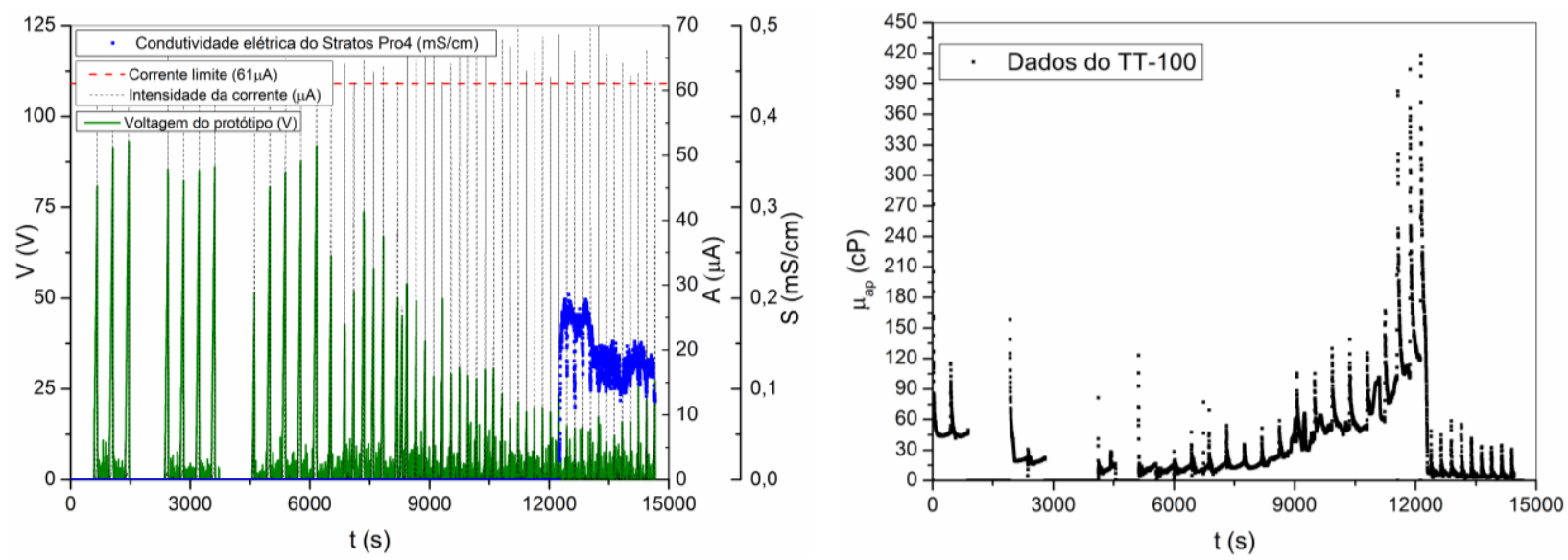

Figura 7 - À esquerda dados de voltagem, intensidade de corrente e condutividade elétrica em função do tempo de teste, à direita dados de viscosidade aparente em função do tempo de teste.

À esquerda da Figura 7 as linhas verdes contínuas representam os picos de tensão (valor de estabilidade elétrica), as linhas pontilhadas pretas os picos de corrente e os pontos em azul a condutividade elétrica do fluido. Percebe-se que à medida que a água entra no sistema, o valor de estabilidade elétrica começa a decrescer, como esperado devido ao aumento da concentração de água na emulsão. Observando à direita, é possível ver o impacto que a entrada de água tem na reologia. À medida que água é adsorvida, a viscosidade aumenta significativamente até o ponto em que sofre uma abrupta queda. Se observado o instante de tempo em que isto ocorre, sendo este transportado de volta para o gráfico da direita, é exatamente no mesmo instante em que a emulsão é rompida, onde o condutivímetro deixa de marcar zero.

\section{CONCLUSÕES}

Vários sensores foram selecionados no mercado para avaliar suas potencialidades em compor uma malha sensorial capaz de fornecer dados de reologia, densidade, estabilidade e condutividade elétrica. Muitos desses sensores não foram especificamente construídos para trabalharem com fluidos de perfuração, portanto alguns necessitaram de modificações e outros foram totalmente desenvolvidos. Os resultados obtidos em processo foram sempre confrontados com resultados laboratoriais aferidos em instrumentos de bancada que são referência para a 
indústria petrolífera. Por meio desta comparação, entende-se que tal automação é possível com desvios de até $20 \%$.

\section{REFERÊNCIAS}

BARNES, H. A., 2000. A Handbook of Elementary Rheology. Institute of Non-Newtonian Fluid Mechanics, University of Wales.

BROOKFIELD INC., 1993. Instruction Manuals and Guides., Model TT-100.

CRAFT, HOLDEN \& GRAVES, 1962. Well Design: Drilling and Production. Prentice - Hall, Inc. Englewood Cliffs, New Jersey.

FANN INSTRUMENTS, 2009, revision C, Part No. 209064, Model 23D Electrical Stability Tester, Instruction Manual.

FANN INSTRUMENTS, 2013, revision G, Part No. 100063065 TRU-WATETM, Fluid Density Balance, Model 141, Instruction Manual.

FANN INSTRUMENTS, 2013, revision N, Part No. 208878, Model 35A Viscometer, Instruction Manual.

GANDElMAN, R.A., MARTINS, A.L., TEIXEIRA, G.T., ARAGÃO, A.F.L., NETO R.M.C., LINS, D.G.M., LENZ, C., GUILARDI P., MARI, A., ESSS, 2013. “Real Time Drilling Data

HALLIDAY D., RESNICK R., WALKER L., 1993. Fundamentos de Física, Volume 3, $4^{\circ}$ Edição, Editora LTC.

ROCHA, L. A. S., JUNQUEIRA, P., ROQUE, J. L., 2003. "Overcoming Deep and Ultra Deepwater Drilling Challenges. Offshore Technology Conference”. DOI:10.4043/15233-MS

Diagnosis Implemented In Deepwater Wells - A Reality”, OTC-24275-MSOTC-24275-MS, Rio de Janeiro, 29 a 31de Outubro, 2013, Brasil.

SANFELD, A., STEINCHEN, A., 2008. "Emulsion Stability, from Dilute to Dense Emulsions Role of Drops Deformation”. Faculdade de Ciências de Saint Jérôme, França. Avanços em ciências de coloides e interface, páginas 1-65, Elsevier.

SCHRAMM, L. L., 2000. Surfactants: Fundamentals and Applications in the Petroleum Industry. Imprensa universitária de Cambridge, Cambridge, 2000. 